\title{
J. Metal-DNA/RNA interaction
}

\section{$\mathbf{J - 0 3}$}

\section{Assembly of programed metal arrays inside the DNA double helix}

Guido Clever', Motoo Kaneko', Kentaro Tanaka², Thomas Carell $^{3}$, Mitsuhiko Shionoya ${ }^{1}$

${ }^{\mathbf{1}}$ Department of Chemistry, Graduate School of Science, The University of Tokyo, Tokyo 113-0033, Japan.

${ }^{2}$ Department of Chemistry, Graduate School of Science, Nagoya University, Nagoya 464-8602, Japan.

${ }^{3}$ Department of Chemistry, Ludwig-Maximilians-University Munich, Germany.guido@chem.s.u-tokyo.ac.jp

Base pairing in natural oligonucleotides relies on hydrogen bonding and p-stacking. Applying coordinative interactions between ligand-like nucleobases and metal cations is a new way of assembling artificial oligonucleotide duplexes. We previously reported the salen metal base pair which is formed when two artificial salicylic aldehyde nucleobases in a DNA duplex are treated with ethylenediamine and a variety of transition metal ions. Addition of 1 eq of $\mathrm{Cu}^{2+}$ increased the melting temperature tremendously by $42^{\circ} \mathrm{C}$. The complexation was examined by UV-, CD- and EPR-spectroscopy and high resolution ESI mass spectrometry. Incorporation of numerous metal-base pairs into oligonucleotides may lead to interesting compounds for nano-technological applications. So far we were able to stack ten manganese or ten copper ions inside the double helix. Furthermore, we prepared sequence specifically arrays of up to five copper ions plus five mercury ions inside one duplex using two ligands with orthogonal selectivity.

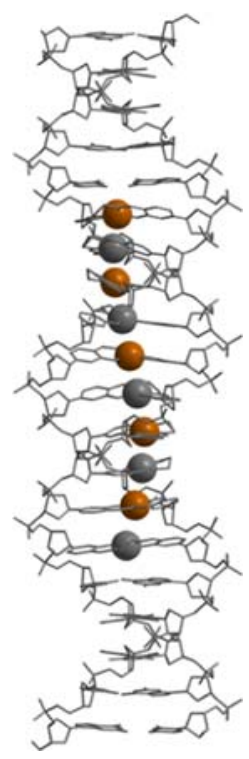

\section{References}

1. Clever GH, Kaul C, Carell T (2007) Angew Chem Int Ed 46:62266236 (review)
2. Clever GH, Polborn K, Carell T (2005) Angew Chem Int Ed 44:7204-7208

3. Tanaka K, Clever GH, Takezawa Y, Yamada Y, Kaul C, Shionoya M, Carell T (2006) Nat Nanotech 1:190-194

\section{J-04}

\section{Characterization of a complex between heme} and G-quadruplex DNA

Y. Yamamoto' ${ }^{1}$, K. Saito ${ }^{1}$, Y. Nakano' ${ }^{1}$, T. Arai ${ }^{1}$, H. Tai ${ }^{1}$, S. Nagatomo ${ }^{1}$, H. Hemmi ${ }^{2}$, H. Mita ${ }^{3}$

${ }^{1}$ Department of Chemistry, University of Tsukuba, Tsukuba 305-8571, Japan, ${ }^{2}$ National Food Research Institute, Tsukuba 305-8642, Japan. ${ }^{3}$ Department of Life, Environment and Materials Sciences, Fukuoka Institute of Technology, Fukuoka 821-0295, Japan. yamamoto@chem.tsukuba.ac.jp

A single repeat sequence of the human telomere, d(TTAGGG), has been shown to form all parallel G-quadruplex DNA in the presence of $\mathrm{K}^{+}$, which dimerizes spontaneously. The size and planarity of a $\mathrm{G}$-quartet are well suited for interacting with a porphyrin ring through $\pi-\pi$ stacking. We have demonstrated the formation of a stable 1:1 complex, called "heme-DNA", between heme (iron(II or III)-protoporphyrin IX complex) and G-quadruplex DNA assembled from d(TTAGGG). In this study, we have characterized structural and ligand binding properties of heme-DNA.

Upon the reduction of heme iron of heme-DNA in the absence and presence of CO gas, UV-Vis absorption spectra of the complexes were remarkably similar to those of deoxy- and CO-forms of myoglobin $(\mathrm{Mb})$, respectively, indicating that heme-DNA and $\mathrm{Mb}$ are highly alike in terms of the heme environments. Diamagnetic $\mathrm{CO}$ form of heme-DNA is stable enough to be characterized by NMR. ${ }^{1} \mathrm{H}$ NMR study revealed that an exchangeable proton signal is resolved at $\sim-3.5 \mathrm{ppm}$. The anomalous upfield shift of the signal may be due to porphyrin ring current effect, and hence is attributable to an axial ligand. The NMR results not only
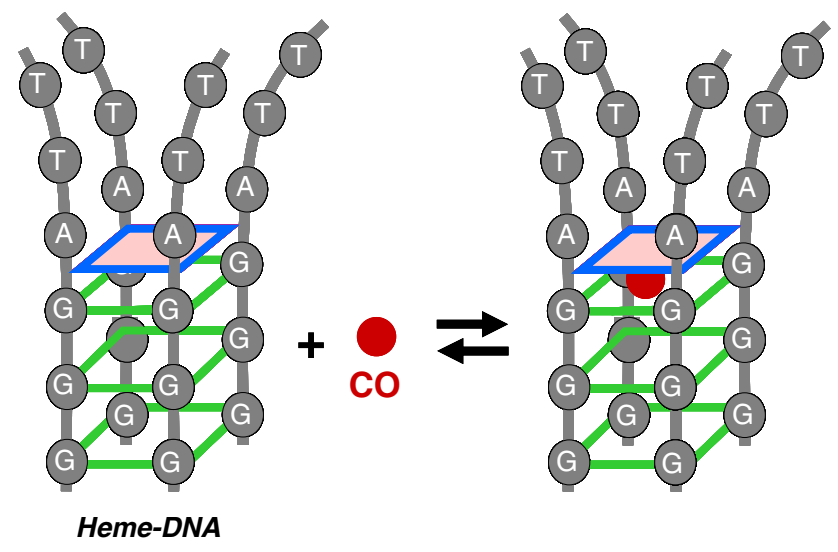

Fig. 1 CO binding of heme-DNA 
confirmed the formation of the coordination bond between heme iron and a DNA base, possibly adenine, but also revealed the formation of the bond between heme iron and exogenous CO.

\section{$\mathrm{J}-05$}

\section{Using artificial nucleosides for the site-specific} functionalization of nucleic acids with metal ions Jens Müller

Westfälische Wilhelms-Universität Münster, Institut für Anorganische und Analytische Chemie, Corrensstr. 28/30, 48149 Münster, Germany. mueller.j@uni-muenster.de

DNA is one of the most prominent molecules currently applied in the construction of nanoscale objects. A site-specific functionalization of these supramolecules with metal ions is possible via the incorporation of artificial nucleosides that comprise ligands with an increased affinity toward selected metal ions [1-3]. These nucleosides can be either appended at the terminus of an oligonucleotide, enabling the formation of metal-mediated cross-links between different duplexes, or in the middle of oligonucleotides, enabling an alignment of metal ions along the helical axis via the formation of metal-mediated base pairs.

This talk will comprise examples for both types of nucleoside incorporation: Attachment of a bipyridine nucleoside to the end of a self-complementary oligonucleotide leads to helices that aggregate only in the presence of suitable metal ions [4]. Moreover, the use of azole nucleosides within an oligonucleotide can be used to generate DNA duplexes with a continuous stack of silver(I) ions. The solution structure of such a helix (see Fig. 1), representing the first structural insight into duplexes with neighboring metal-mediated base pairs, will be reported [5].

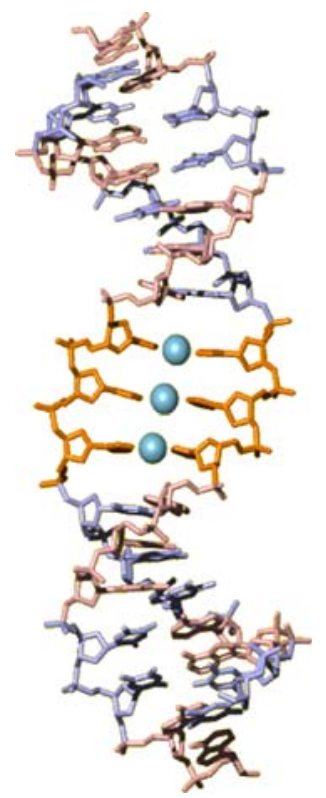

\section{References}

1. Müller J (2008) Eur J Inorg Chem 3749

2. Tanaka K, Shionoya M (2007) Coord Chem Rev 251:2732

3. Clever GH, Kaul C, Carell T (2007) Angew Chem Int Ed 46:6226

4. Düpre N, Welte L, Gómez- Herrero J, Zamora F, Müller J (2009) Inorg Chim Acta 362:985

5. Johannsen S, Düpre N, Böhme D, Sigel RKO, Müller J (in preparation)

\section{J-06}

DNA as supramolecular scaffold for functional molecules

Tom Bandy, Ashley Brewer, Jon Burns, Simon Gerrard, Gabriella Marth, ThaoNguyen Nguyen, Eugen Stulz

School of Chemistry, University of Southampton, Highfield, Southampton SO17 1BJ, UK. est@ soton.ac.uk

We have established a general synthetic route to porphyrin-nucleosides and their subsequent site specific incorporation into oligonucleotides to create multiporphyrin arrays [1]. Up to eleven consecutive porphyrins could be incorporated into DNA giving access to a multiporphyrin array of approximately $10 \mathrm{~nm}$ in length. The spectroscopic data and structure calculations indicate the formation of a stable helical array in the single strand porphyrin-DNA. The $\pi$-stack of the porphyrins leads to strong electronic interaction between the chromophores. The stability and spectroscopic features of the porphyrin arrays will be discussed. A new zipper array with induced stability and energy transfer properties has recently been realized [2]. Further structural properties arising from synchrotron $\mathrm{CD}$ spectroscopy data will be presented. We have also expanded our system to terpy-containing nucleotides and porphyrindinucleotides which can form further supramolecular structures through metal complexation and branching.
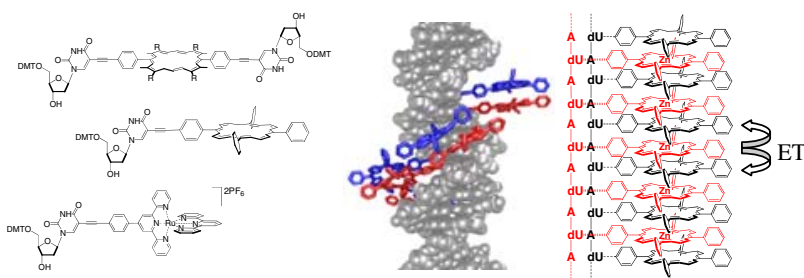

\section{References}

1. Fendt LA, Bouamaied I, Thöni S, Amiot N, Stulz E (2007) J Am Chem Soc 129:15319-15329

2. Nguyen T, Brewer A, Stulz E (2009) Angew Chem Int Ed 48:1974-1977

\section{J-07}

Coexistence of four conformers, including an elusive conformer, in a model for the N7-Pt-N7 crosslink adduct formed by $\mathrm{Pt}$ anticancer drugs and $\mathrm{d}(\mathrm{GpG})$ Vidhi Maheshwari, Patricia A. Marzilli, Luigi G. Marzilli* Department of Chemistry, Louisiana State University, Baton Rouge, LA 70803, USA.1marzil@1su.edu

$\mathbf{L P t}\left(\mathrm{d}\left(\mathrm{G}^{*} \mathrm{pG}^{*}\right)\right.$ models for platinum anti-cancer drug DNA intrastrand crosslink adducts are too dynamic to allow characterization of the four possible conformers $\left(G^{*}=N 7\right.$-platinated $G$ residue $)$. Models with $\mathbf{L}=$ a bidentate ligand bulkier than those found in active drugs have a slower interchange rate, allowing clear identification of three (HH1, HH2, and $\Delta \mathrm{HT} 1$ ) conformers (the guanine bases have either a head-to-head $(\mathrm{HH})$ or a head-to-tail $(\mathrm{HT})$ orientation, and the sugar-phosphodiester backbone has a normal (1) or opposite (2) propagation direction. We hypothesized that $\mathbf{L}$ bulk destabilizes the fourth conformer $(\Lambda \mathrm{HT} 2)$, that a Goldilocks-like $\mathbf{L}$ with just enough bulk to slow $\mathrm{G}^{*}$ rotation would not prevent formation of this elusive fourth conformer, and that the $\mathbf{R}_{\mathbf{4}} \mathbf{d t}$ (bis-3,3'-(5,6-dialkyl-1,2,4triazine), $\mathbf{R}=\mathrm{Me}$ and $\mathrm{Et}$ ) ligands have suitable bulk. NMR and HPLC data indicated that $\left(\mathbf{R}_{\mathbf{4}} \mathbf{d t}\right) \operatorname{Pt}\left(\mathrm{d}\left(\mathrm{G}^{*} \mathrm{pG} \mathrm{G}^{*}\right)\right)$ adducts do form the $\Lambda \mathrm{HT} 2$ conformer in addition to the now well-known HH1, HH2 and $\Delta \mathrm{HT} 1$ conformers. Unlike the $\left(\mathbf{R}_{\mathbf{4}} \mathbf{d t}\right) \operatorname{Pt}\left(5^{\prime}-\mathrm{GMP}\right)_{2}$ adducts, the $\left(\mathbf{R}_{\mathbf{4}} \mathbf{d t}\right)$ 
$\operatorname{Pt}\left(\mathrm{d}\left(\mathrm{G}^{*} \mathrm{p} \mathrm{G}^{*}\right)\right)$ adducts did not give EXSY peaks, a result providing clear evidence that the sugar-phosphodiester backbone slows conformer interchange. Indeed, the $\Lambda \mathrm{HT} 2$ conformer formed and converted to other conformers extremely slowly, allowing us to interpret HPLC data to indicate that the $\Lambda \mathrm{HT} 2$ conformer formed from $\mathrm{HH} 2$ and not from HH1. Bulkier $\mathbf{L}\left(\mathbf{E t}_{\mathbf{4}} \mathbf{d t}\right.$ vs. $\left.\mathbf{M e}_{\mathbf{4}} \mathbf{d t}\right)$ decreased the abundance of the $\Lambda \mathrm{HT} 2$ conformer, supporting the hypothesis that steric crowding disfavors this conformer. The effects of bulk and the slow kinetics of formation explain why an abundant $\Lambda \mathrm{HT} 2$ conformer was not found previously.

\section{$\mathrm{J}-\mathbf{0 8}$}

\section{Is there anything unique about the ammonia ligands} of cis- and Transplatin?

\section{Bernhard Lippert}

Fakultät Chemie, Technische Universität Dortmund, 44221 Dortmund, Germany. bernhard.lippert@tu-dortmund.de Cisplatin, cis- $\mathrm{PtCl}_{2}\left(\mathrm{NH}_{3}\right)_{2}$, and likewise its trans-isomer, are small molecules, consisting of merely 11 atoms. The question may be asked, if anything else but the Pt center and the difference in spatial orientation of their ligands is responsible for their surprising (albeit differential) biological chemistry. It has long been anticipated that the $\mathrm{NH}_{3}$ ligands in the two compounds are chemically inert, but this is not generally true. In this lecture, possible roles and observed reactivity patterns of the $\mathrm{NH}_{3}$ ligands in these compounds will be summarized and discussed. They will include the following aspect:

- Hydrogen bonding of $\mathrm{Pt}-\mathrm{NH}_{3}$ groups to nucleobases (model compounds vs. DNA adducts),

- Directed hydrogen bonding of $\mathrm{Pt}-\mathrm{NH}_{3}$ to anions [1],

- Loss of $\mathrm{NH}_{3}$ ligands,

- Deprotonation of $\mathrm{NH}_{3}$ ligands during condensation reactions with other metal entities [2] as well as with organic molecules [3].

\section{References}

1. Yin L, Shen W-Z, Sanz Miguel PJ, Lippert B (submitted)

2. Gil Bardají E, Freisinger E, Costisella B, Schalley CA, Brüning W,

Sabat M, Lippert B (2007) Chem Eur J 13:6019-6039

3. Lippert B et al (2009) (to be submitted)

\section{J-09}

\section{Non-covalent DNA binding properties of novel supramolecular ruthenium metallo-drugs \\ Anna Lęczkowska, Michael J. Hannon*}

School of Chemistry, University of Birmingham, Edgbaston, Birmingham B15 2TT, UK. A.leczkowska@bham.ac.uk,

m.j.hannon@bham.ac.uk

Selective interaction of small molecules with nucleic acids is of great interest, because of its considerable potential in anticancer and gene therapy. We have recently reported cationic metallosupramolecular dinuclear helicates that target the biologically relevant form of DNA acting through non-covalent interactions. These compounds with a size and shape similar to protein zinc fingers bind strongly to the major groove of the DNA, causing bending and intramolecular DNA coiling [1] and more interestingly, target DNA three-way junctions fitting perfectly into the junction point of the three double helical arms [2,3]. In addition, the compounds exhibit significant cytotoxic activity against cancer cell lines; however, they do not show genotoxicity [4]. We are currently developing inert, tetracationic, coordinatively saturated and unsaturated dinuclear ruthenium helicates with different structural topologies and investigating their effect on DNA structures. The DNA binding properties and cytotoxic activity of these compounds, as well as the effect of chirality of our helicates on the DNA binding affinity will be presented.

\section{References}

1. Hannon MJ (2007) Pure Appl Chem 79:2243-2261

2. Oleksi A, Blanco AG, Boer R, Usón I, Aymamí J, Roger A, Hannon MJ, Coll M (2006) Angew Chem Int Ed 45:1227-1231

3. Cerasino L, Hannon MJ, Sletten E (2007) Inorg Chem 46:6245-6251

4. Pascu GI, Hotze ACG, Sanchez-Cano C, Kariuki BM, Hannon MJ (2007) Angew Chem Int Ed 46:4374-4378

\section{J-10}

Platinum coordination compounds with anticancer properties: study of DNA binding inspires new drug design

Jan Reedijk

Leiden Institute of Chemistry, Leiden University, P.O. Box, 9502, 2300 RA, Leiden, The Netherlands. reedijk@chem.leidenuniv.nl Metal coordination compounds, especially those of $\mathrm{Pt}$ and $\mathrm{Ru}$, that have metal-ligand exchange rates comparable to cell-division processes, often are highly active as anticancer agents. The classical compound cis-diamminedichloridoplatinum(II), (cisplatin), and many derivatives are known to bind to DNA. Studying the binding of such platinum compounds to nucleic acids could result in the development of new DNA-binding drugs [1]. Using molecular mechanistic knowledge, strategies for the design of new metal-containing drugs were developed. Special attention has been given to apply bifunctional DNA binding of such new compounds. Examples of new insights into the mechanism of action will be presented, including the use of other metals that bind to DNA, like $\mathrm{Cu}$ and $\mathrm{Ru}$ [2]. Multifunctional DNA binding of such compounds, allows the use of a fluorescent group to follow the process of the compounds online while in the cells [3]. Combination of Cu-DNA binding [4] with platinum, has resulted in a new compounds to be used for both DNA cutting [5] and for new anticancer chemistry [6].

\section{References}

1. Reedijk J (2003) Proc Natl Acad Sci USA 100:3611-3616

2. van der Schilden K, Garcia F, Kooijman H, Spek AL, Haasnoot JG, Reedijk J (2004) Angew Chem Int Ed 43:5668-5670

3. Kalayda GV, Jansen BAJ, Wielaard P, Tanke HJ, Reedijk J (2005)

J Biol Inorg Chem 10:305-315

4. Maheswari PU, Roy S, den Dulk H, Barends S, van Wezel G, Kozlevcar B, Gamez P, Reedijk J (2006) J Am Chem Soc 128:710 711

5. Özalp-Yaman S, de Hoog P, Amadei G, Pitié M, Gamez P, Dewelle J, Mijatovic T, Meunier B, Kiss R, Reedijk J (2008) Chem Eur J 14:3418-3426

6. Reedijk J (2008) Plat Metals Rev 52:2-11

\section{J-11}

\section{Design of metal anticancer complexes with unusual} DNA interactions

\section{Peter J. Sadler}

Department of Chemistry, University of Warwick, Coventry CV4

7AL, UK. P.J.Sadler@warwick.ac.uk

Metal complexes which induce conformational distortions and other damage in DNA may exhibit anticancer activity as a consequence of 
protein recognition and further downstream-processing We have designed inert diazido Pt(IV) prodrugs which are relatively non-toxic to cells until activated by light. Particularly potent is the complex trans, trans, trans- $\left[\mathrm{Pt}(\right.$ pyridine $\left.)\left(\mathrm{NH}_{3}\right)(\mathrm{OH})_{2}\left(\mathrm{~N}_{3}\right)_{2}\right]$, which targets $\mathrm{G}$ residues on DNA and produces DNA damage which is less readily repaired than that produced by cisplatin [1].

Certain organometallic half-sandwich $\mathrm{Ru}(\mathrm{II})$ arene complexes [(arene) $\mathrm{Ru}(\mathrm{XY}) \mathrm{Z}]$, where $\mathrm{XY}=$ an $N, N$ - or $N, O$ - or $O, O$-chelating ligand, exhibit anticancer activity in vitro and in vivo [2]. Particularly potent are complexes with extended arenes which can intercalate into DNA [3]. Curiously the Os(II) analogs appear to cause less DNA bending but more marked DNA unwinding [4]. I will also discuss new methods for exploring metal-induced shape changes in DNA.

We thank the EPSRC, MRC, EC (Marie Curie and COST), and Science City (AWM) for support, and our collaborators.

\section{References}

1. Mackay FS, Woods JA, Heringová P, Kaspárková J, Pizarro AM, Moggach SA, Parsons S, Brabec V, Sadler PJ (2007) Proc Natl Acad Sci USA 104:20743-20748

2. Yan Y-K, Melchart M, Habtemariam A, Sadler PJ (2005) Chem Commun 4764-4776

3. Liu H-K, Berners-Price SJ, Wang F, Parkinson JA, Xu J, Bella J, Sadler PJ (2006) Angew Chem Int Ed 45:8153-8156

4. Kostrhunova H, Florian J, Novakova O, Peacock AFP, Sadler PJ, Brabec V (2008) J Med Chem 51:3635-3643

\section{$\mathrm{J}-12$}

\section{Addition of osmium to DNA pyrimidine bases} Akiko Nomura, Kazuki Tainaka, Kazuo Tanaka, Tadashi Umemoto, Akimitsu Okamoto

RIKEN Advanced Science Institute, Wako, Saitama 351-1098, Japan. aki-okamoto@riken.jp

Nucleic acids often acquire new functions by forming a variety of complexes with metal ions. Osmium, in an oxidized state, also reacts with C5-methylated pyrimidines. However, the control of the sequence specificity of osmium complexation with DNA is still immature, and the worth of the resulting complexes is unknown. We designed a bipyridine-attached adenine derivative for sequence-specific osmium complexation. Sequence-specific osmium complexation was achieved by the hybridization of a short DNA molecule containing this functional nucleotide to a target DNA sequence, and resulted in the formation of a crosslinked structure. The interstrand crosslink clearly distinguished methylated cytosines from unmethylated ones, which was used to quantify the degree of methylation at a specific cytosine in a whole genome. This is a conceptually new prototype for methylation typing.

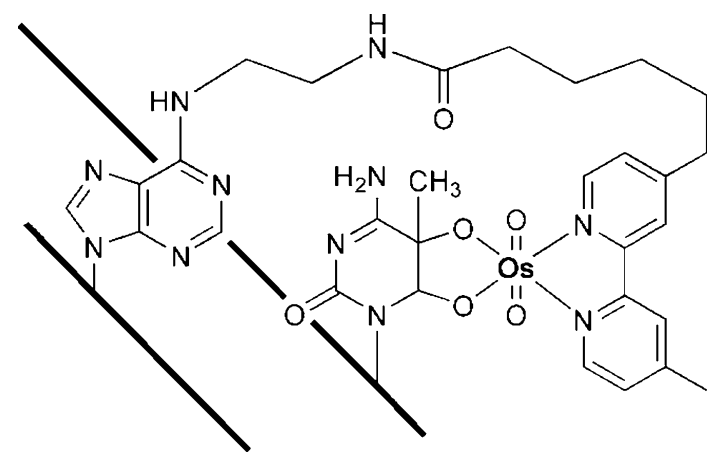

\section{J-13}

Artificial restriction DNA cutter for gene manipulation Makoto Komiyama

Research Center for Advanced Science and Technology, University of Tokyo, Tokyo, 153-8904, Japan.

komiyama@mkomi.rcast.u-tokyo.ac.jp

Current molecular biology is based on site-selective scission of DNA by restriction enzymes. However, site-specificity of naturally occurring restriction enzymes is too low to manipulate huge DNA. In order to solve this problem, we recently developed man-made tools which cut double-stranded DNA at desired site (artificial restriction DNA cutter; ARCUT) [1]. These tools are composed of (1) Ce(IV)/EDTA complex as molecular scissors and (2) two pseudo-complementary PNAs (blue lines in Fig. 1). By changing the sequences and the lengths of PNA strands, the scission-site and site-specificity are freely chosen.

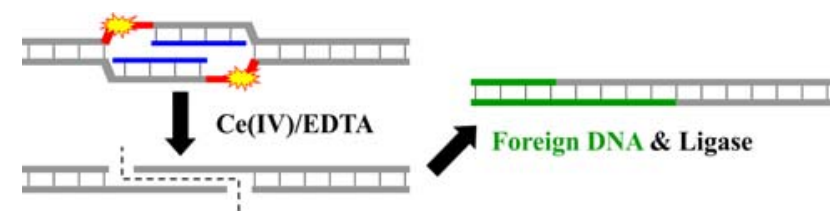

Fig. 1 Site-selective scission of double-stranded DNA by ARCUT and ligation of the scission fragment with foreign DNA

With the use of ARCUT, even huge DNAs (e.g., the whole genome of human beings) were selectively cut at target site. The resultant scission fragments were ligated with foreign DNAs (green lines in Fig. 1) and successfully expressed in mammalian cells. Applications of ARCUT for promotion of homologous recombination in human cells are also presented.

\section{References}

1. Komiyama M, Aiba Y, Ishizuka T, Sumaoka J (2008) Nat Protocols $3: 646-655$

\section{J-14}

Modifications on the corrin ring of coenzyme $B_{12}$ lead to altered structural switches of the $b t u B$ riboswitch of $E$. coli

Sofia Gallo, Stefan Mundwiler, Roger Alberto, Roland K. O. Sigel Institute of Inorganic Chemistry, University of Zürich, 8057 Zurich, Switzerland.sgallo@aci.uzh.ch

Riboswitches are short and highly conserved sequences within mRNAs that regulate gene expression. Thereby direct interaction between the riboswitch and a specific metabolite leads to a structural rearrangement of the RNA and hence to an altered expression of the downstream genes [1]. We are studying the coenzyme $\mathrm{B}_{12}$ binding to the $b t u B$ riboswitch of $E$. coli which regulates the expression of the outer membrane $\mathrm{B}_{12}$ transporter. Earlier studies demonstrated the corrin ring system of the $\mathrm{B}_{12}$ molecule to be the crucial part to induce the structural change of this riboswitch. The presence of the two axial ligands instead significantly increases the binding affinity [2].

Here, we describe how alterations of the corrin ring side-chains influence the binding between the $\mathrm{B}_{12}$ derivatives and the btuB RNA. The role of the individual sidechains was investigated by applying acid-modified vitamin $B_{12}$ derivatives [3] in in-line probing experiments, i.e. structure dependent self-cleavage of the RNA sequence to map conformational changes. Our results show that these $\mathrm{B}_{12}$ derivatives carrying a carboxylic acid moiety induce either no structural 
change or lead to a completely different RNA architecture compared to coenzyme $B_{12}$ and vitamin $B_{12}$. The amide side chains are thus crucial for the correct rearrangement of the btuB riboswitch.

Financial support by the Swiss National Science Foundation, the University of Zürich, and within the COST Action D39 is gratefully acknowledged.

\section{References}

1. Serganov A, Patel DJ (2007) Nat Rev Genet 8:776-790

2. Gallo S, Oberhuber M, Sigel RKO, Kräutler B (2008) ChemBioChem 9:1408-1414

3. Spingler B, Mundwiler S, Ruiz-Sánchez P, van Staveren DR, Alberto R (2007) Eur J Inorg Chem 2641-2647

\section{J-15}

\section{Splitting single molecules into two distinct} subpopulations: the effect of $\mathrm{Ca}^{2+}$ coordination on single group II intron ribozymes

\section{Roland K. O. Sigel}

Institute of Inorganic Chemistry, University of Zürich,

Winterthurerstrasse 190, 8057 Zurich, Switzerland.

roland.sigel@aci.uzh.ch

Formation of the three-dimensional architecture of large RNAs is achieved by $\mathrm{M}^{2+}$ binding, i.e. usually $\mathrm{Mg}^{2+}$ [1]. The so-called D135 ribozyme, derived from the self-splicing yeast mitochondrial group II intron Sc.ai $5 \gamma$, folds stepwise to the active and most compact state $\mathrm{N}$. No kinetic traps exist, only on-pathway intermediates are touched, and $\mathrm{N}$ is only reached transiently from a near native state $\mathrm{F}$ [2]. D135 is highly sensitive to trace amounts of $\mathrm{Ca}^{2+}$, whose binding leads to a drastic loss in activity [3]. Here we investigate the effect of $\mathrm{Ca}^{2+}$ on the folding pathway on the single molecule level by fluorescence experiments (smFRET). The binding of $\mathrm{Ca}^{2+}$ leads to the formation of two distinct subpopulations of molecules, which do not interchange: Whereas population 1 folds similarly to the $\mathrm{Mg}^{2+}$-only bound molecules, in population 2 , the most compact state is highly populated but misfolded. This unprecedented behavior can be explained by specific binding pockets for $\mathrm{Ca}^{2+}$ and cooperative binding of $\mathrm{Ca}^{2+}$ to the ribozyme [4].

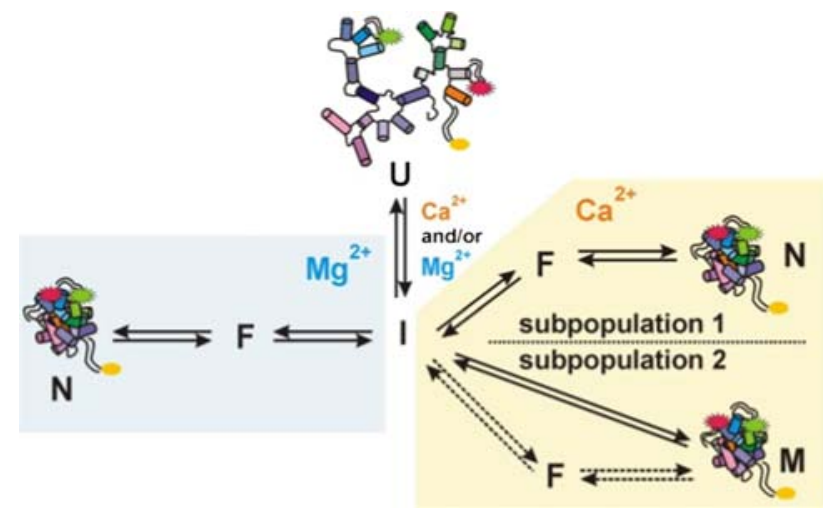

Financial support by the Swiss National Science Foundation, the University of Zürich and within the COST D39 Action is gratefully acknowledged.

\section{References}

1. Freisinger E, Sigel RKO (2008) Coord Chem Rev 105:1385313858

2. Steiner M, Karunatilaka KS, Sigel RKO, Rueda D (2008) Proc Natl Acad Sci USA 105:13853-13858

3. Erat MC, Sigel RKO (2008) J Biol Inorg Chem 13:1025-1036

4. Steiner M, Rueda D, Sigel RKO (submitted)

\section{J-16}

RNA Metallobiochemistry: ribozyme active sites and metallo-drugs

Victoria J. DeRose, W. Luke Ward, Alethia Hostetter, Erich Chapman

Department of Chemistry, Institute of Molecular Biology, University of Oregon, Eugene, OR 97403-1253, USA. derose@uoregon.edu

The RNA biopolymer folds into complex structures that control diverse activities in gene expression. We are applying spectroscopic and biochemical methods to investigate the influence of metal ions on RNA folding and chemical activity, with a focus on the unique combined nucleobase-metal ion mechanism of the hammerhead ribozyme. In combination with kinetic studies, the coordination properties of site-bound metal ions are probed using EPR, NMR, and other spectroscopic techniques. Site-directed spin labeling (SDSL) methods allow the influence of these ions on RNA folding to be deciphered. In complementary studies, we are also investigating the binding properties of metal-based therapeutic agents with the diverse cellular structures of RNA. Time permitting, initial kinetic, structural, and in vivo investigations of RNA as a target for the anticancer compound cisplatin will be presented.

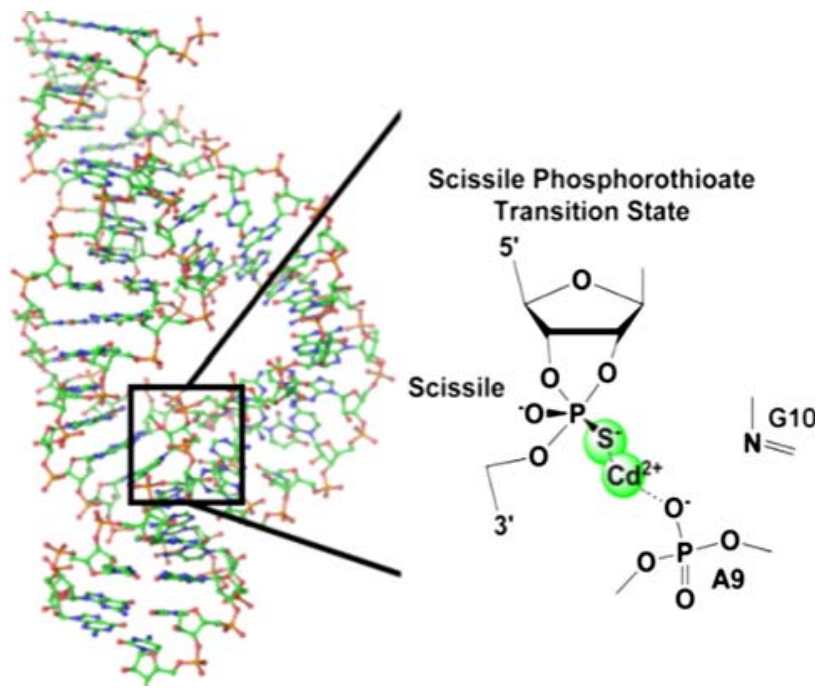

\section{References}

1. Vogt M, Lahiri S, Hoogstraten CG, Britt RD, DeRose VJ (2006) J Am Chem Soc 129:16764-16770

2. Osborne EM, Ruehle M, Ward WL, DeRose VJ (submitted)

3. Hostetter AA, Chapman EG, DeRose VJ (submitted) 